\section{Has inductive probability been proved impossible?}

SIR-The interesting letter by Popper and Miller $^{1}$ and the subsequent correspondence $^{2-4}$ leaves in some doubt the question whether a proof of the impossibility of inductive probability been given. As this would be a remarkable achievement, it is no criticism of these authors that we raise this question again. In our view the answer is a clear "No". We shall follow your other correspondents in not burdening the discussion with references to extensive earlier literature.

The key elements in the Popper-Miller (PM) argument are: first the evidence $e$, when found, counter-supports the implication $h \leftarrow e$ of a hypothesis $h$ by the evidence; and second, this is due to the negative nature of that part of the support for $h \leftarrow e$ arising from $e$ which is not purely deductive. From this type of consideration, PM infer the impossibility of inductive probability. In both the original PM and the following discussion, the part played by the negation $\bar{e}$ was not considered explicitly. Yet it is helpful to do so in showing what is going on in these arguments, as will now be explained. Two basic results, to be formulated as "theorems", are useful preludes to this explanation.

\section{Theorem A}

$$
\begin{aligned}
p(h \leftarrow e \mid e) & \leqslant p(h \leftarrow e \mid e)+p(\bar{h} \mid e) p(\bar{e}) \\
& =p(h \leftarrow e) \leqslant p(h \leftarrow e \mid \bar{e})=1 \\
p(h \vee e \mid \bar{e}) & \leqslant p(h \vee e \mid \bar{e})+p(\bar{h} \mid \bar{e}) p(e) \\
& =p(h \vee e) \leqslant p(h \vee e \mid e)=1
\end{aligned}
$$

Theorem B The following have the same sign:

$$
\begin{aligned}
p(h \mid e)-p(h) & =\underline{p(h \mid e) p(\bar{e})}-p(h \mid \bar{e}) p(\bar{e}) \\
& =p(\bar{h} \mid \bar{e}) p(\bar{e})-\underline{p(\bar{h} \mid e) p(\bar{e})} \\
& =p(\bar{h})-p(\bar{h} \mid e)
\end{aligned}
$$

and

$$
\begin{aligned}
& p(\bar{h} \mid \bar{e})-p(\bar{h})=p(\bar{h} \mid \bar{e}) p(e)-p(\bar{h} \mid e) p(e) \\
& =p(h \mid e) p(e)-p(h \mid \bar{e}) p(e) \\
& =p(h)-p(h \mid \bar{e}) \text {. }
\end{aligned}
$$

Theorem A shows the origin of theorem 1 of PM that

$$
p(h \leftarrow e)>p(h \leftarrow e \mid e) \quad(\text { if } p(e) \neq 1)
$$

as follows. The underlined terms are positive, so that (4) could be incorporated in (1). Note that $p(h \leftarrow e)=p(h \vee \bar{e})$ allows for the possibility of $\bar{e}$, which possibility is ruled out by the assumption that $e$ has occurred. This makes the inequality (4) intuitive as a logical necessity (which is incidentally independent of any inferential relation between $e$ and $h$ ).

Since $h \leftarrow e \equiv h \vee \bar{e}, \bar{e}$ must "support" $h \leftarrow e$ maximally, or $p(h \leftarrow e \mid \bar{e})=1$. It is intuitively to be expected, therefore, that $e$ "counter supports" $h \leftarrow e$. These statements interpret Theorem A (an extension of theorems 1 and 2 of PM).

Theorem B shows relations between four "support" functions whose definition

$$
s(h, e)=p(h / e)-p(h)
$$

was introduced into the correspondence by Jeffrey ${ }^{3}$. They are shown as differences in different ways. Adopting the nomenclature of your correspondents (which is possibly misleading), we call the underlined terms "non-deductive". The introduction of negations is again helpful here, for they show, using (5), that the "non-deductive" part in (2) appears with a positive sign (support) in $s(h, e)$, but with a negative sign (counter support) in $s(\bar{h}, e)$. Similarly for (3). Thus the remark by PM that all probabilistic support is purely deductive requires some different definition of these terms or some restriction on their interpretation.

Theorem B also tells us something very reasonable about the function (5):

$$
s(h, e) \geqslant 0 \text { implies } s(h, \bar{e}) \leqslant 0,
$$$$
s(\bar{h}, e) \leqslant 0, s(\bar{h}, \bar{e}) \geqslant 0
$$

while $s(h, e) \leqslant 0$ implies $s(h, \bar{e}) \geqslant 0$,

$$
s(\bar{h}, e) \geqslant 0, s(\bar{h}, \bar{e}) \leqslant 0
$$

In words, if $e$ supports $h$ then it countersupports $\bar{h}$, and also $\bar{e}$ counter-supports $h$ but supports $\bar{h}$. The converse is given by (7).

The above considerations explain, we hope, some of the results obtained by PM, by your correspondents and by us. They do not really go beyond basic facts such as: $h \vee e$ follows deductively from $e, h \vee \bar{e}$ follows deductively from $\bar{e}$, together with probability algebra. In that sense, they are obtained by strict deduction and have nothing to do with induction. It is for these reasons that we have to give the answer "No" to the question in the title.

$$
\text { J. WISE }
$$

$$
\text { P. T. LANDSBERG }
$$

\section{University of Southampton}

Southampton SO9 $5 \mathrm{NH}, \mathrm{UK}$

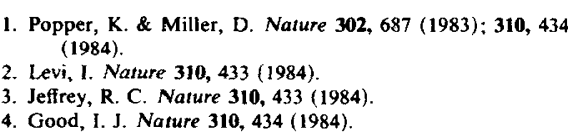
(1984).

2. Levi, I. Nature 310, 433 (1984)

3. Jeffrey, R. C. Nature 310, 433 (1984)

4. Good, I. J. Nature 310, 434 (1984).

POPPER AND MILLER REPLY-In 1878, Peirce drew a sharp distinction between "explicative, analytic, or deductive" and "amplifiative, synthetic, or (loosely speaking) inductive" reasoning. He characterized the latter as reasoning in which "the facts summed up in the conclusion are not among those stated in the premisses". This was the background to our brief letters (refs 1, 2 in Wise and Landsberg's letter). We identified the amplifiative part, relative to any evidence $e$, of any hypothesis $h$, with the conditional proposition $h \leftarrow e$; and we proved quite ○)1985 Nature Publishing Group generally that if $p(h, e) \neq 1 \neq p(e)$, then

$$
s(h \leftarrow e, e)=-c t(h, e) c t(e)<0
$$

where

$$
s(x, y)=p(x, y)-p(x)
$$

and

$$
c t(x, y)=1-p(x, y) .
$$

Accordingly, the ("amplifiative") part of the hypothesis $h$ that goes beyond the evidence $e$ is always counter-supported by the evidence. This was our thesis. (The other part, which we wrote $h \vee e$, is of course a deductive consequence of $e$, and is deductively supported by $e$.) Both the measures of support and of content are additive:

$$
\begin{aligned}
& s(h, z)=s(h \vee e, z)+s(h \leftarrow e, z) \\
& c t(h, z)=c t(h \vee e, z)+c t(h \leftarrow e, z)
\end{aligned}
$$

(Equations (2) and (3) are valid for all $h$, $e$ and $z$, and thus for $z=e$.) Wise \& Landsberg note that there are other ways of writing $s(h, e)$ in the form $\ulcorner \pm s(x, e)+ \pm s(y, e)\urcorner$; for example

$$
s(h, e)=-s(\bar{h} \vee e, e)+-s(\bar{h} \leftarrow e, e)
$$

This equation (4) is a simplification of their formula (2). It can be obtained by substituting $\bar{h}$ for $h$ in our (2) above, and multiplying by -1 . Obviously, neither $\bar{h} \vee e$ nor $\vec{h} \leftarrow e$ is part of the content of $h$. Thus we fail to see how our (4) - that is to say, their (2)-can possibly challenge our thesis, or have any bearing whatsoever on it.

Fallowfield, Manor Close, KARL POPPER

Penn, High Wycombe,

Buckinghamshire HP10 8HZ, UK DAVID Miller

Department of Philosophy,

University of Warwick,

Coventry CV4 7AL, UK

\section{Gastric cancer and salivary nitrate and nitrite}

SIR - Forman et al. ${ }^{1}$ reported lower salivary and nitrite levels in healthy subjects from areas of Great Britain with high gastric cancer incidences, compared with subjects from areas of that country with low incidences of this cancer. These unexpected findings were used to argue against the theor $y^{2,3}$ that the intra-gastric formation of carcinogenic $N$-nitroso compounds (NOC) from nitrite is involved in the aetiology of gastric cancer. Although this theory remains controversial, I have three objections to the suggestion that the results presented by Forman et al. disprove the NOC theory.

(1) A lower nitrate intake was reported ${ }^{1}$ in the diet of the high-risk subjects, which accounted for their lower levels of salivary nitrate and nitrite. Since most dietary nitrate (76-89\% in this study) arises from vegetables, which supply much of our dietary vitamin $C$, the high-risk subjects 